\title{
Dehydrin expression in drought-stressed tall fescue
}

C.A. GUERBER, C.P. WEST, R.D. CARSON and A.M. HAVELY 1366 W. Altheimer Dr., University of Arkansas, Fayetteville, AR 72704 USA

cguerber@uark.edu

\begin{abstract}
Tall fescue (Lolium arundinaceum) drought tolerance is often enhanced by endophyte infection; however, the physiological mechanisms are not clearly understood. Endophyte-infected $(\mathrm{E}+)$ and endophyte-free (E-) clones of two contrasting tall fescue genotypes were drought stressed to investigate differences in endophyte-mediated dehydrin expression. Both genotypes had shown enhanced drought survival due to their endophyte in previous trials. As drought stress intensified, E+ and E- plants of both genotypes produced dehydrins; however, quantitative and qualitative differences were noted. Genotype 60, a mesic type, accumulated more dehydrins in E+ than in E- plants, whereas genotype 330, a xeric type, displayed low levels of dehydrin and no differences between E+ and E- plants in accumulation patterns. Lack of dehydrin response in genotype 330 suggests that putative dehydrin involvement in endophyte-enhanced tiller survival may not be a universal mechanism in tall fescue. In the absence of endophyte, genotype 330 still expressed much less dehydrin than E- genotype 60, indicating lesser importance of dehydrin in a xeric plant for dehydration tolerance, perhaps benefiting more from constitutive adaptive traits in the host.

Keywords: dehydrin, drought, tall fescue
\end{abstract}

\section{Introduction}

Tall fescue [Lolium arundinaceum (Schreb. Darbysh.] has a mutualistic relationship with the endophytic fungus Neotyphodium coenophialum (Morgan-Jones \& Gams) Glenn, Bacon \& Hanlin, enabling it to withstand periods of summer drought while being grazed. Dehydrins are a conspicuous soluble protein in plant

Figure 1 Accumulation of dehydrins in genotype 60 tall fescue at (a) Stage 1, (b) Stage 2, and (c) Stage 3 of drought stress. Lanes indicate three replicate extractions per treatment.

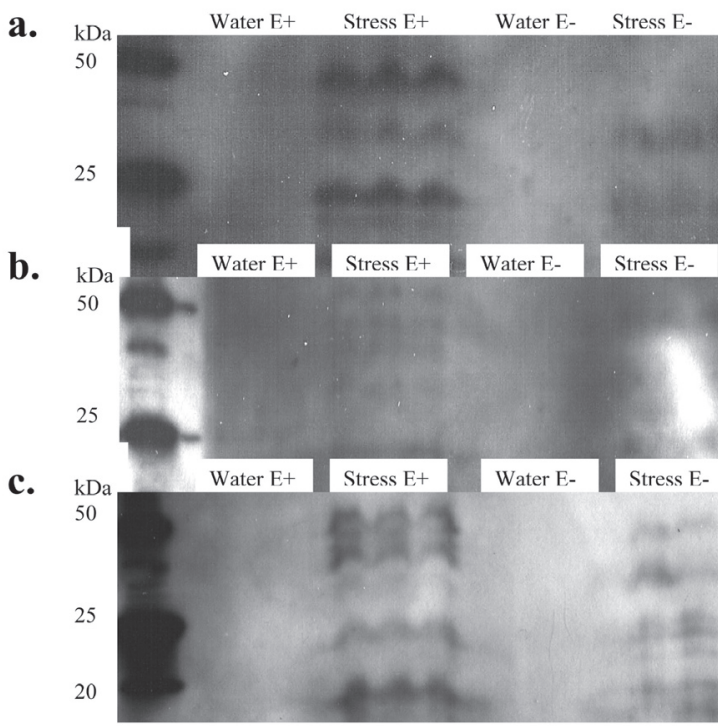

organs subjected to dehydration and osmotic stress (Close 1996) and are therefore putatively associated with protective mechanisms that may aid in survival of such organs. They accumulate in areas of the plasma membrane where dehydration is more detrimental (Cellier 1998). It has been hypothesised that dehydrins function by stabilising large-scale hydrophobic interactions (Koag et al. 2003) and transcription machinery (Allagulova et al. 2003). Tall fescue cultivars can now be inoculated with endophyte strains lacking mammalian toxins to impart protective advantages to the grass (Bouton et al. 2002). Identifying traits such as dehydrins in nontoxic endophytes that are highly associated with host drought tolerance would expedite the selection of optimal symbionts. Our objective was to investigate whether the endophyte promotes dehydrin accumulation in drought stressed tall fescue.

\section{Methods}

Tall fescue genotypes 60 and 330 were selected to investigate the influence of naturally occurring endophytes on the expression of drought-induced dehydrin proteins. Genotype 60 is a mesic type plant morphologically adapted to humid environments, with wide, flagging leaves and relatively thin cuticle. Its phenotype and ploidy (hexaploid $-2 n=6 x=42$ ) are similar to the commonly grown 'Kentucky-31' cultivar. Genotype 330, a decaploid (2n

Figure 2 Accumulation of dehydrins in genotype 330 tall fescue at (a) Stage 1, (b) Stage 2, and (c) Stage 3 of drought stress. Two replicate extractions per treatment.

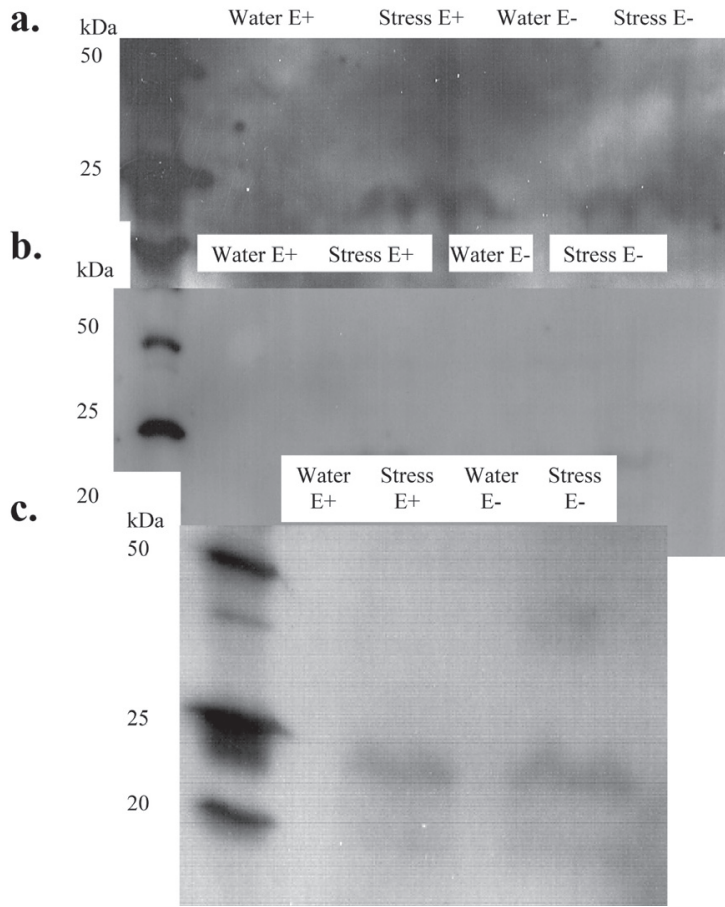


$=10 \mathrm{x}=70$ ), is a xeric-type plant morphologically adapted to arid climates, with narrow, upright leaves and thick cuticle. Genetically identical replicate plants from each genotype were propagated from single parental clones. Both genotypes contained their respective natural wild-type endophytes $(\mathrm{E}+)$, which were genetically distinct from each other (unpublished data). Carson (2004) demonstrated that these endophytes significantly enhanced tiller drought survival in both host genotypes. Endophyte-free (E-) clones of 60 and 330 were produced by treating E+ plants with the fungicide propiconizol [1-(2-(2-4-dichlorophenyl)-4propyl-1,3-dioxolan-2-yl) methyl-1H-1,2,4-triazole; Tilt ${ }^{\mathbb{R}}$, CibaGeigy, Agricultural Division, Greensboro, NC, USA]. Endophyte absence was verified microscopically.

\section{Experimental design and tissue sampling}

Endophyte-infected and E- clones of genotypes 60 and 330 were grown in loamy fine sand in white plastic dishpans in a greenhouse in a split plot arrangement. The pan served as the whole unit designated as a water stress treatment by genotype combination, with endophyte infection status split within each pan. The split treatment consisted of alternating rows of $\mathrm{E}+$ plants and E- plants with five plants in each row and a total of four rows per pan. Two replicate pans were maintained as well-watered controls, while two replicate pans per drought stage treatment were allowed to dry. Samples were taken at three morphologically defined stages of water deficit: Stage 1 - beginning of leaf rolling, Stage 2 - beginning of leaf desiccation, and Stage 3 - inner leaf blade desiccated. The bottom $2 \mathrm{~cm}$ of each tiller base was sampled by cutting at the apical meristem and removing the outer leaf sheaths, but retaining the sheath of the youngest, fully expanded leaf.

\section{Protein extraction, electrophoresis, and western hybridisation}

Dehydrins of the DHN4 class were detected using western hybridisation (Close et al. 1993). Total protein was extracted by freezing tissue samples in liquid nitrogen and homogenising in extraction buffer (Anderson et al. 1992). The supernatant was desalted in PD-10 desalting columns (Amersham Biosciences, Piscataway, NJ.). Total protein was fractionated by $15 \%$ SDSPAGE cell electrophoresis at 200 volts for $1 \mathrm{~h}$. Separated polypeptides were blotted onto $0.45-\mu \mathrm{m}$ PVDF filter membrane by electrophoresis. Western hybridisation consisted of blocking the membranes in $0.2 \%$ non-fat dried milk for $1 \mathrm{~h}$, incubating them for $1 \mathrm{~h}$ with an antidehydrin polyclonal antibody at a dilution of 1:10,000 in phosphate-buffered saline (PBS) and then for $1 \mathrm{~h}$ with the secondary antibody, goat anti-rabbit fragment at a dilution of 1:25,000 in PBS-TWEEN. The dehydrin/antibody immunological reaction was detected using the Bio-Rad Immunstar ${ }^{\mathrm{TM}}$ chemiluminescence system.

\section{Results}

Dehydrin proteins were detected in E+ and E- plants of both genotypes during all three stages of drought stress, and as water deprivation was prolonged, dehydrin expression intensified (Figs. 1 and 2). No dehydrin was expressed in any of the well-watered controls. In stressed E+ plants of genotype 60 , seven bands were recognised at Stage 3 (Fig. 1c.) by the antidehydrin antibody at $52,50,48,35,24,22$ and $18 \mathrm{kDa} \mathrm{M}_{\mathrm{r}}$ (relative migration). The 50,24 and $18 \mathrm{kDa}$ dehydrins were upregulated at Stage 1 and 2 (Fig. 1a, b), and in Stage 3, 52 and $48 \mathrm{kDa}$ dehydrins were also strongly accumulated (Fig. 1c). There was an unexplainably weak chemiluminescent signal at Stage 2 (Fig. 1b.). In stressed
E- plants, only five bands were recognised at 48, 35, 24, 22 and $18 \mathrm{kDa} \mathrm{M}$ with lower copy numbers than in the E+ plants, indicating that the endophyte stimulated dehydrin expression in response to drought (Fig. 1). Genotype $330 \mathrm{E}+$ and E- clones produced dehydrins at only 24 and $20 \mathrm{kDa} \mathrm{M}_{\mathrm{r}}$, with no difference due to endophyte in dehydrin accumulation under drought stress (Fig. 2).

\section{Discussion}

The two genotypes exhibited different patterns of dehydrin expression in the presence and absence of the endophyte in response to progressive drought stress, even though both genotypes had previously exhibited greater tiller survival during drought in $\mathrm{E}+$ than in E- clones (Carson 2004). The xeric genotype, which has constitutive morphological adaptations to drought stress (e.g. thick cuticles, narrow upright leaves), accumulated dehydrins with a low number of forms (bands), at low copy number, and similarly in the presence and absence of its endophyte. Removing endophyte from the mesic genotype reduced the number of dehydrin bands and in lower copy number. This result was obtained in two more repeats of the same trial. Endophyte enhancement of tiller survival was associated with previously reported (Carson 2004) promotion of dehydrin expression in genotype 60 , but not in genotype 330 . Dehydrin production in genotype 60 may or may not have contributed directly to tiller base survival, and it likely did not in genotype 330. Endophyteenhanced tiller survival in genotype 330 probably depends on other biochemical protective mechansisms. This result suggests that the physiological modes involved in tiller survival are variable and complex among grass-Neotyphodium symbiota, and that selection based on a single biochemical trait may not be effective.

The regulation of dehydrin expression is part of the early response to water deficit in many plant species. In cocksfoot (Dactylis glomerata L.), an association between low-molecular weight (22 to $26 \mathrm{kDa}$ ) dehydrins and drought survival has been documented by Volaire (2002), suggesting that dehydrins aid in promoting dehydration tolerance. Shen et al. (2004) reported high levels of dehydrin expression in the desiccation-tolerant plant Boea crassifolia. Jiang \& Huang (2002) found dehydrins in two turf-type cultivars of tall fescue, 'Southeast' and 'Rebel Jr.', in which a greater accumulation of dehydrins under drought stress was associated with reduced dehydration damage. Their study examined accumulation of dehydrins in fully extended leaves, but did not investigate any endophyte effects.

In conclusion, our results indicate 1) genotypic variation between hosts in dehydrin expression independently of endophyte infection, and 2) differential endophyte effect on response to drought stress by two tall fescue genotypes with respect to dehydrin expression. The xeric genotype 330, which has morphological constitutive defenses, accumulated dehydrins at low levels equally well in the presence or absence of the endophyte, whereas the mesic genotype 60 accumulated more dehydrins at higher copy numbers in the presence of the endophyte. Further study incorporating a greater number of genotypes is required to determine whether dehydrins are indeed involved in summer tiller survival and whether genomic analysis of endophytes can identify biochemical markers associated with enhanced host survival in novel symbiota. 


\section{ACKNOWLEDGEMENTS}

We are indebted to Dr. Timothy Close, University of California-Riverside, for providing dehydrin antibody, and to the USDA-ARS Dale Bumpers Small Farm Research Center for support through Specific Cooperative Agreement no. 6227-21310-007-21S.

\section{REFERENCES}

Allagulova, C.R.; Gimalova, F.R.; Shakirova, F.M.; Vakhitov, V.A. 2003. The plant dehydrins: structure and putative functions. Biochemistry(Moscow) 68: 945-951.

Anderson, J.V.; Chevone, B.I.; Hess, J.L. 1992. Seasonal variation in the antioxidant system of eastern white pine needles. Plant Physiology 98: 501-508.

Bouton, J.G.; Latch, G.C.M.; Hill, N.S.; Hoveland, C.S.; McCann, M.A.; Watson, R.H.; Parish, J.A.; Hawkins, L.L.; Thompson, F.N. 2002. Reinfection of tall fescue cultivars with non-ergot alkaloid producing endophytes. Agronomy Journal 93: 567-574.

Carson, R.D. 2004. Biochemical responses of tall fescue to endophyte infection during water deficit. M.Sc. thesis. University of Arkansas Library.
Cellier, F.; Conejero, G.; Brietler, J-C; Casse, F. 1998. Molecular and physiological responses to water deficit in droughttolerant and drought-sensitive lines of sunflower. Plant Physiology 116: 319-328.

Close, T.J.; Fenton, R.D.; Moonan, F. 1993. A view of plant dehydrins using

antibodies specific to the carboxyl terminal peptide. Plant Molecular Biology 23: 279-286.

Close, T.J. 1996. Dehydrins: emergence of a biochemical role of a family of plant dehydration proteins. Physiologium Plantarum 97: 795-803.

Jiang, Y.; Huang, B. 2002. Protein alterations in tall fescue in response to drought stress and abscisic acid. Crop Science 42: 202-207.

Koag, M.C.; Fenton, R.D.; Wilkens, S.; Close; T.J. 2003. The binding of maize DHN1 to lipid vesicules. Gain of structure and lipid specificity. Plant Physiology 131: 1-8.

Shen, Y.; Tang, M. J.; Hu, Y. L.; Lin, Z. P. 2004. Isolation and characterization of dehydrin-like gene from drought-tolerant Boea crassifolia. Plant Science 166: 1167-1175.

Volaire, F. 2002. Drought survival, summer dormancy and dehydrin accumulation in contrasting cultivars of Dactylis glomerata. Physiologia Plantarum 116: 42-51. 